Journal for ImmunoTherapy of Cancer

\title{
challenging populations: recommendations of the Advisory Committee of Spanish Melanoma Group (GEM)
}

Maria Gonzalez-Cao (1) , ${ }^{1}$ Teresa Puertolas, ${ }^{2}$ Mar Riveiro, ${ }^{3}$ Eva Muñoz-Couselo, ${ }^{4}$ Carolina Ortiz, ${ }^{4}$ Roger Paredes, ${ }^{5,6}$ Daniel Podzamczer, ${ }^{7}$ Jose Luis Manzano, ${ }^{8}$ Jose Molto, ${ }^{5,6}$ Boris Revollo, ${ }^{5,6}$ Cristina Carrera, ${ }^{9}$ Lourdes Mateu, ${ }^{5,6}$ Sara Fancelli, ${ }^{1,10}$ Enrique Espinosa, ${ }^{11}$ Bonaventura Clotet, ${ }^{5,6}$ Javier Martinez-Picado, ${ }^{5,12}$ Pablo Cerezuela, ${ }^{13}$ Ainara Soria, ${ }^{14}$ Ivan Marquez-Rodas (1), ${ }^{15}$ Mario Mandala $\left(\mathbb{C},{ }^{16}\right.$ Alfonso Berrocal, ${ }^{17}$ for the Spanish Melanoma Group (GEM)

To cite: Gonzalez-Cao M, Puertolas T, Riveiro M, et al. Cancer immunotherapy in special challenging populations: recommendations of the Advisory Committee of Spanish Melanoma Group (GEM). Journal for ImmunoTherapy of Cancer 2021;9:e001664. doi:10.1136/ jitc-2020-001664

Accepted 31 January 2021
Check for updates

(C) Author(s) (or their employer(s)) 2021. Re-use permitted under CC BY-NC. No commercial re-use. See rights and permissions. Published by BMJ.

For numbered affiliations see end of article.

Correspondence to Dr Maria Gonzalez-Cao; mgonzalezcao@oncorosell.com

\section{ABSTRACT}

Cancer immunotherapy based on the use of antibodies targeting the so-called checkpoint inhibitors, such as programmed cell death-1 receptor, its ligand, or CTLA-4, has shown durable clinical benefit and survival improvement in melanoma and other tumors. However, there are some special situations that could be a challenge for clinical management. Persons with chronic infections, such as HIV-1 or viral hepatitis, latent tuberculosis, or a history of solid organ transplantation, could be candidates for cancer immunotherapy, but their management requires a multidisciplinary approach. The Spanish Melanoma Group (GEM) panel in collaboration with experts in virology and immunology from different centers in Spain reviewed the literature and developed evidence-based guidelines for cancer immunotherapy management in patients with chronic infections and immunosuppression. These are the first clinical guidelines for cancer immunotherapy treatment in special challenging populations. Cancer immunotherapy in chronically infected or immunosuppressed patients is feasible but needs a multidisciplinary approach in order to decrease the risk of complications related to the coexistent comorbidities.

\section{INTRODUCTION}

Therapy with immune checkpoint inhibitors that block inhibitory receptors of effector lymphocytes mainly programmed cell death-1 (PD-1) and Cytotoxic T-Lymphocyte Antigen 4 (CTLA-4) or their ligand interactions (PD-L1) is now the standard treatment for different types of cancers. However, data on the feasibility of using these therapies in patients with special clinical situations, such as chronic infections or immunosuppression, are scarce. HIV-1 infection, viral hepatitis, and previous solid organ transplantation are common exclusion criteria in most cancer clinical trials due to concerns about potential complications and the arguable balance between expected benefits and risks. Due to the relevant clinical benefit of immune checkpoint blockers in different types of cancer, including melanoma, development of evidence-based guidelines for the management of these patients is relevant. These guidelines are not exclusively focused on melanoma treatment.

\section{METHODS}

A multidisciplinary panel of experts in medical oncology, dermatology, immunology and infectious diseases developed these clinical practice guidelines for the management of approved cancer immunotherapy drugs (anti-PD-1/PD-L1 and anti-CTLA-4 antibodies) in 'special clinical situations' related to HIV-1 infection, viral hepatitis, tuberculosis (TB) infection and solid organ transplantation. The authors developed the guidelines based on a critical review of literature, including case series, case reports and clinical trials, as well as their own observational data (online supplemental table S1). These guidelines are not intended to be a systematic review of the literature, but rather to develop a recommendation based on judgment of experts. Panelists discussed the final recommendations in a multidisciplinary cancer 
conference that was held in Barcelona on January 24, 2020 (https://www.groupgem.es/eventos-2020/). Due to the limitations of the available evidence, the guidelines are non-mandatory recommendations. Levels of evidence and grade of recommendation were classified according to the European Society for Medical Oncology guidelines (https://www.esmo.org/ Guidelines/ESMO-Guidelines-Methodology).

\section{HIV-1 INFECTION Incidence and pathophysiology}

Since the onset of the HIV/AIDS pandemic in 1981, 74.9 million people have contracted HIV. AIDS-related illnesses account for 32 million deaths. In 2018, 37.9 million people were living with HIV (PLHIV) globally, 1.7 million people became newly infected, and 770,000 people died from AIDS-related illnesses (Joint United Nations Programme on HIV and AIDS Fact Sheet 2019). Life expectancy of PLHIV under viral suppressive combination antiretroviral therapy (cART) approaches that of non-infected persons, but the state of chronic inflammation prompts them to a higher risk of cancer and other comorbidities. ${ }^{1}$ This is the reason why cancer is now one of the leading causes of death in HIV-1-infected persons. ${ }^{2}$ Patients infected with HIV-1 have been excluded from clinical trials that evaluate cancer immunotherapies, ${ }^{3}{ }^{4}$ but recently two prospective trials have shown the safety and feasibility of these therapies in PLHIV (online supplemental table S1). ${ }^{5}{ }^{6}$ Previously, retrospective series and case reports had suggested similar safety and activity of anti-PD-1/ PD-L1 antibodies in PLHIV, compared with uninfected individuals. ${ }^{7-9}$ Grade 3 adverse events ranged from $0 \%$ to $20 \%$, and grade 4 adverse events appeared in less than $1 \% \cdot{ }^{56}$ Also, antitumoral activity of anti-PD-1/PD-L1 antibodies in PLHIV was demonstrated, with a disease control rate of $17 \%-50 \% .{ }^{5}$ In the prospective studies, all patients included had undetectable plasma viral load (pVL) under cART. Moreover, throughout the anti-PD-1/ PD-L1 treatment, pVL remained undetected and $\mathrm{CD} 4^{+}$ and $\mathrm{CD}^{+} \mathrm{T}$ cell counts were stable. Regarding baseline $\mathrm{CD}^{+} \mathrm{T}$ cell counts, most PLHIV on cART recover $\mathrm{CD} 4^{+} \mathrm{T}$ cell counts, but some individuals with delayed initiation of cART or who have received previous immunosuppressive therapies, such as chemotherapy, never recovered normal $\mathrm{CD}^{+} \mathrm{T}$ cell levels. The clinical trials led by Uldrick et $a t^{t}$ and Gonzalez-Cao et a $a \tilde{\varphi}$ included six patients and one patient, respectively, with baseline $\mathrm{CD}^{+} \mathrm{T}$ cells below 200 cells per $\mathrm{mm}^{3}$, without suffering any complications due to immunotherapy. Interestingly, one subject with a previously treated metastatic non-small cell lung cancer included in the DURVAST trial with baseline $\mathrm{CD} 4^{+} \mathrm{T}$ cell counts of 164 cells per $\mathrm{mm}^{3}$ had a long-lasting complete response $(32+$ months $) .{ }^{510}$ Case reports also support the safety of treating patients with $\mathrm{CD} 4^{+} \mathrm{T}$ cell counts lower than 100 cells per $\mathrm{mm}^{3} .^{11}$

\section{Management and monitoring}

Screening of HIV-1 infection previous to administration of immunotherapy is not mandatory, except for patients with AIDS-defining cancers or persons of groups at higher risk of HIV-1 infection, such as sex workers, men who have sex with men, or intravenous drug users (level of evidence III, grade of recommendation A).

HIV-1-infected individuals with tumor types that are candidates for immunotherapy must be considered for cancer treatment (level of evidence III, grade of recommendation A). Pretreatment pVL must be checked. Immunotherapy should be administered when pVL is undetectable (level of evidence III, grade of recommendation A). Patients must continue on cART therapy (level of evidence III, grade of recommendation A). It is not necessary to modify their cART when they start anticancer treatment with PD-1/PD-L1 antibodies if there is an effective viral suppression (level of evidence III, grade of recommendation $\mathrm{C}$ ). Blips, defined as transitory detectable HIV viral load below 400 copies/mL, are frequent and have no clinical significance, so it is not necessary to modify anti-PD-1/PD-L1 therapy when blips are detected during therapy (level of evidence III, grade of recommendation A). In patients with recent HIV-1 diagnosis, cART should be started prior to treatment with immunotherapy. Anti-PD-1/PD-L1 treatment should be delayed until viral suppression is achieved (level of evidence IV, grade of recommendation $\mathrm{C}$ ). Viral suppression is generally achieved in most patients after 4 weeks on cART. In cases where anticancer treatment is an emergency, a simultaneous initiation of immunotherapy with cART could be considered, evaluating risk and benefits of every case, although there is no evidence of the safety of this procedure (level of evidence $\mathrm{V}$, grade of recommendation C). For starting anti-PD-1/PD-L1 therapy, pretreatment $\mathrm{CD} 4^{+} \mathrm{T}$ cell counts should preferably be above 200 cells per $\mathrm{mm}^{3}$ (level of evidence III, grade of recommendation B). ${ }^{3}$ Patients with a $\mathrm{CD} 4{ }^{+} \mathrm{T}$ cell counts below 100 cells per $\mathrm{mm}^{3}$ have not been included in clinical trials of anti-PD-1/ PD-L1 antibodies. Therefore, while low CD $4^{+}$ $\mathrm{T}$ cell counts are not an absolute contraindication to immunotherapy, careful evaluation of the risk to benefit ratio in these cases should be assessed (level of evidence $\mathrm{V}$, grade of recommendation $\mathrm{C}$ ) (figure 1).

The preferred anti-PD-1 antibody under this condition would be pembrolizumab due to availability of concordant prospective and retrospective data demonstrating its safety and activity ${ }^{6}$ (level of evidence III, grade of recommendation B). Other anti-PD-1 antibodies have been used in this context with good tolerance, such as nivolumab $^{12}$ (level of evidence IV, grade of recommendation C). The preferred anti-PD-L1 antibody would be durvalumab because its safety has been demonstrated in one clinical trial ${ }^{5}$ (level of evidence III, grade of recommendation $\mathrm{B})$. There are no prospective data on the safety and activity of other checkpoint inhibitors or combinations in PLHIV, but case reports and series of patients treated with ipilimumab or the combination of 


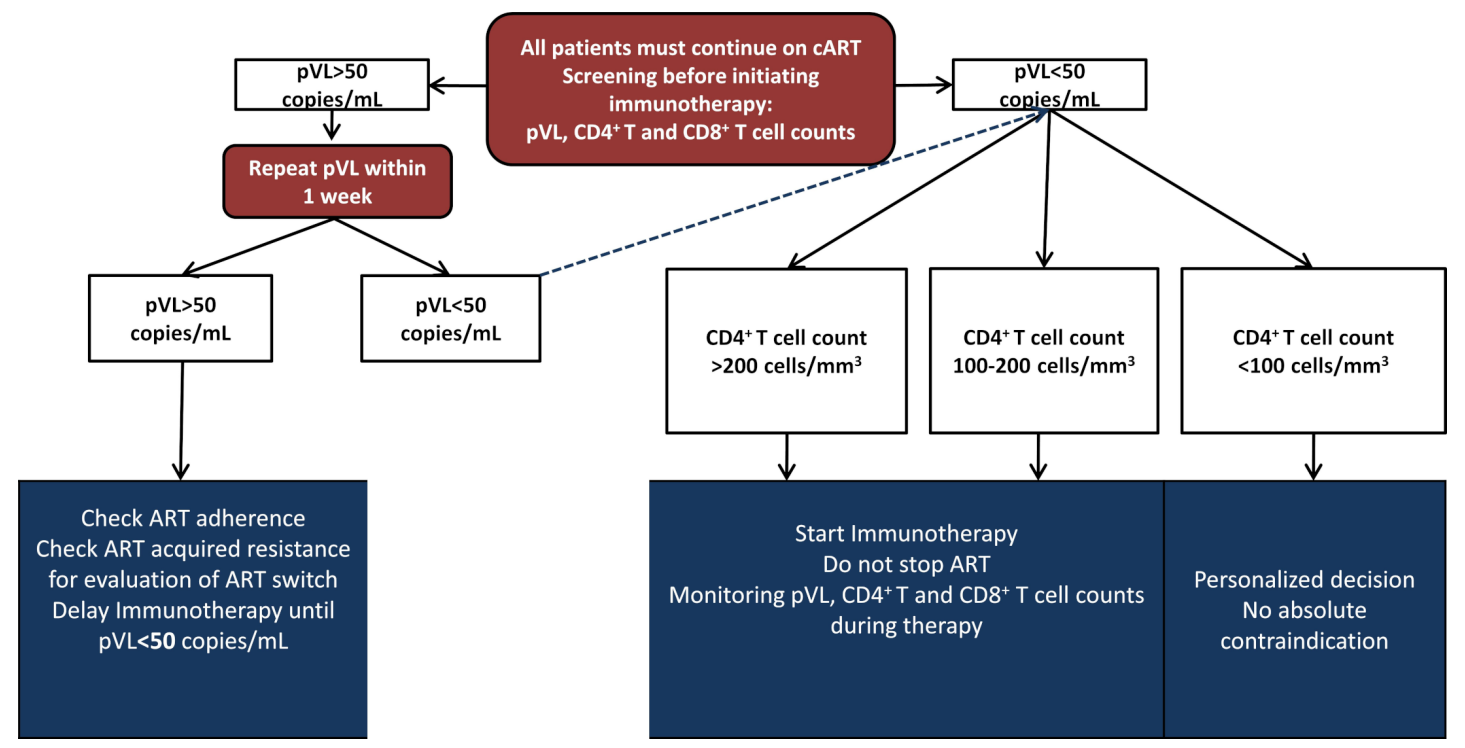

Figure 1 Diagram of management of HIV-1-infected patient candidates for immunotherapy. ART, antiretroviral therapy; cART, combination antiretroviral therapy; pVL, plasma viral load.

ipilimumab plus nivolumab ${ }^{78}$ suggest their safety (level of evidence IV, grade of recommendation C). Other clinical trials are ongoing in order to check the safety of other checkpoint inhibitors in PLHIV, but the mechanisms of action of these therapies do not preclude any significant difference in tolerance and activity among the studied antibodies in this population.

Plasma viremia and $\mathrm{CD} 4^{+}$and $\mathrm{CD} 8^{+} \mathrm{T}$ cell counts must be periodically monitored (every 2 or 3 months) during immunotherapy (level of evidence III, grade of recommendation B). If $\mathrm{pVL}$ becomes detectable in several consecutive analyses, drug resistance genotypic testing and/or therapeutic drug monitoring should be considered (figure 1).

\section{VIRAL HEPATITIS}

\section{Incidence and pathophysiology}

Worldwide, an estimated 257 million and 71 million people are living with chronic hepatitis B (HBV) and hepatitis $\mathrm{C}(\mathrm{HCV})$ viral infection, respectively. ${ }^{13}$ It should be kept in mind that the rate of chronic hepatitis $\mathrm{B}$ (hepatitis B surface antigen (HBsAg)-positive) is relatively low in most high-income countries $(0.5 \%-2 \%)$; however, the percentage of subjects with evidence of resolved $\mathrm{HBV}$ infection (HBsAg-negative with anti-HBc-positive) is $15 \%-20 \% .{ }^{14}$ Although both viruses share the same ways of transmission and can produce chronic infection, there are multiple differences between HCV and HBV pathophysiology. First, HBV, but not HCV, integrates into the human DNA, so when an immunosuppressed person gets infected, HBV could be reactivated, even in cases with an apparently resolved infection (HBsAg-negative with anti-HBc-positive). Second, the clinical evolution and dynamics of the viral infection are clearly different, suggesting that immune response dynamics are much more complex in HBV infection than in the case of HCV.
While HCV infection evolution is predictable, in the case of HBV the clinical evolution is heterogeneous between patients. After HCV infection, some patients develop chronic viremia, and others have a completely resolved infection into the first 6 months after exposure. ${ }^{15}$ After this phase, most chronic HCV-infected patients slowly progress to severe liver disease after more than 20 years, with few exceptions. ${ }^{15}$ In comparison, HBV infection evolves in a much more heterogeneous and unpredictable way throughout its entire course. In chronic HBV infection, there are different stages with different levels of viral replication, immune response and liver function. ${ }^{15}$ Finally, the third important difference between these two viruses is that HCV infection is curable with highly effective oral direct-acting antiviral agents (DAA) ${ }^{16}$ but there is no curative therapy for $\mathrm{HBV}$ (online supplemental data 1 and table S2).

Treatment with immune checkpoint inhibitors in patients with chronic hepatitis deserves special consideration as it could produce complications due to an impairment in hepatic function in the case of an advanced liver disease, but also due to a reactivation of chronic infections. Feasibility of treatment with anti-PD-1/PD-L1 antibodies in patients with cirrhosis was assessed in clinical trials studying the efficacy of anti-PD-1 for patients with hepatocellular carcinoma (HCC). Most patients with HCC included in phase I/II studies of nivolumab, ${ }^{17}$ pembrolizumab $^{18}$ or durvalumab ${ }^{19}$ had compensated cirrhosis without unexpected drug-related adverse events. Few patients with decompensated cirrhosis have been treated with immunotherapy, but data suggest that treatment is also well tolerated. ${ }^{20}$ From an overall perspective, monoclonal antibodies could be considered safe enough even in moderate hepatic insufficiency as liver metabolism is not necessary for clearance. 
Concerning the risk of viral reactivation, recent studies have demonstrated a high rate of viral reactivation of $\mathrm{HBV}$ when these patients are treated with anti-PD-1 antibodies (online supplemental table S1). The most important study included $114 \mathrm{HBsAg}$-positive patients treated with an antiPD-1 antibody for a median time of 18 weeks, showing a reactivation rate of $17 \%$. When patients were simultaneously under treatment with antiviral therapy, the viral reactivation rate decreased to $1.2 \% .{ }^{21}$ Other studies have also demonstrated in viral suppressed HBeAg-negative patients that checkpoint blockade is well tolerated without adverse events, and even immunotherapy was suggested to have some antiviral activity since HBsAg levels declined in most patients. ${ }^{22}$ A recent publication has also found that treatment with anti-PD-1 antibodies with simultaneous treatment with antiviral drugs is safe and precludes viral reactivation. ${ }^{23}$ Indeed, general guidelines for immunosuppressed patients recommend antiviral prophylaxis for patients with occult HBV infection (HBsAg-negative, antiHBc-positive, with detectable HBV DNA) ${ }^{24}$ although the risk of reactivation is very low, ${ }^{2526}$ and that periodic monitoring of alanine aminotransferase levels, HBsAg and HBV DNA should be performed, with antiviral therapy deferred until confirmation of viral reactivation (seroconversion to HBsAg-positive or detectable HBV DNA). The most frequent antiviral drugs used for HBV are nucleoside or nucleotide analogs (NUCs), such as entecavir, tenofovir disoproxil or tenofovir alafenamide. Another consideration to be aware of is the case of development of immune-related adverse events during cancer immunotherapy. Patients treated with high-dose corticosteroids have a moderate risk of HBV reactivation, especially for cases on therapy for more than 4 weeks or when corticosteroids are combined with antitumor necrosis factor (anti-TNF) agents. ${ }^{24}$

In cases of HCV infection, anti-PD-1/PD-L1 and antiCTLA-4 antibodies are also safe (online supplemental table S1). There is no viral reactivation, and moreover in some cases there is a decline in viral replication. A clinical trial of tremelimumab, an anti-CTLA- 4 antibody, in a cohort of 20 patients with HCC revealed a drop in HCV RNA in $75 \%$ of subjects. More recently, the anti-PD- 1 antibody nivolumab demonstrated infection transient reductions in HCV RNA in 60 patients with HCC with HCV infection, although none achieved sustained virological suppression. ${ }^{17}$

In case of HCV infection, treatment with DAA should be based on the risk of progression of liver disease to HCC, according to the grade of liver fibrosis or cirrhosis, and the potential drug interactions.

\section{Management and monitoring}

All patients should be screened for hepatitis viral infection before starting immunotherapy (level of evidence III, grade of recommendation B).

Diagnosis of HCV infection is based on the presence of anti-HCV antibodies and HCV RNA in cases tested positive for anti-HCV. In patients who are HCV RNA-positive, it is mandatory to assess the severity of the liver disease using non-invasive markers in order to rule out advanced liver fibrosis or cirrhosis. In case of liver cirrhosis, liver ultrasound is recommended to exclude HCC, as well as gastroscopy in case of signs of portal hypertension (figure 2).

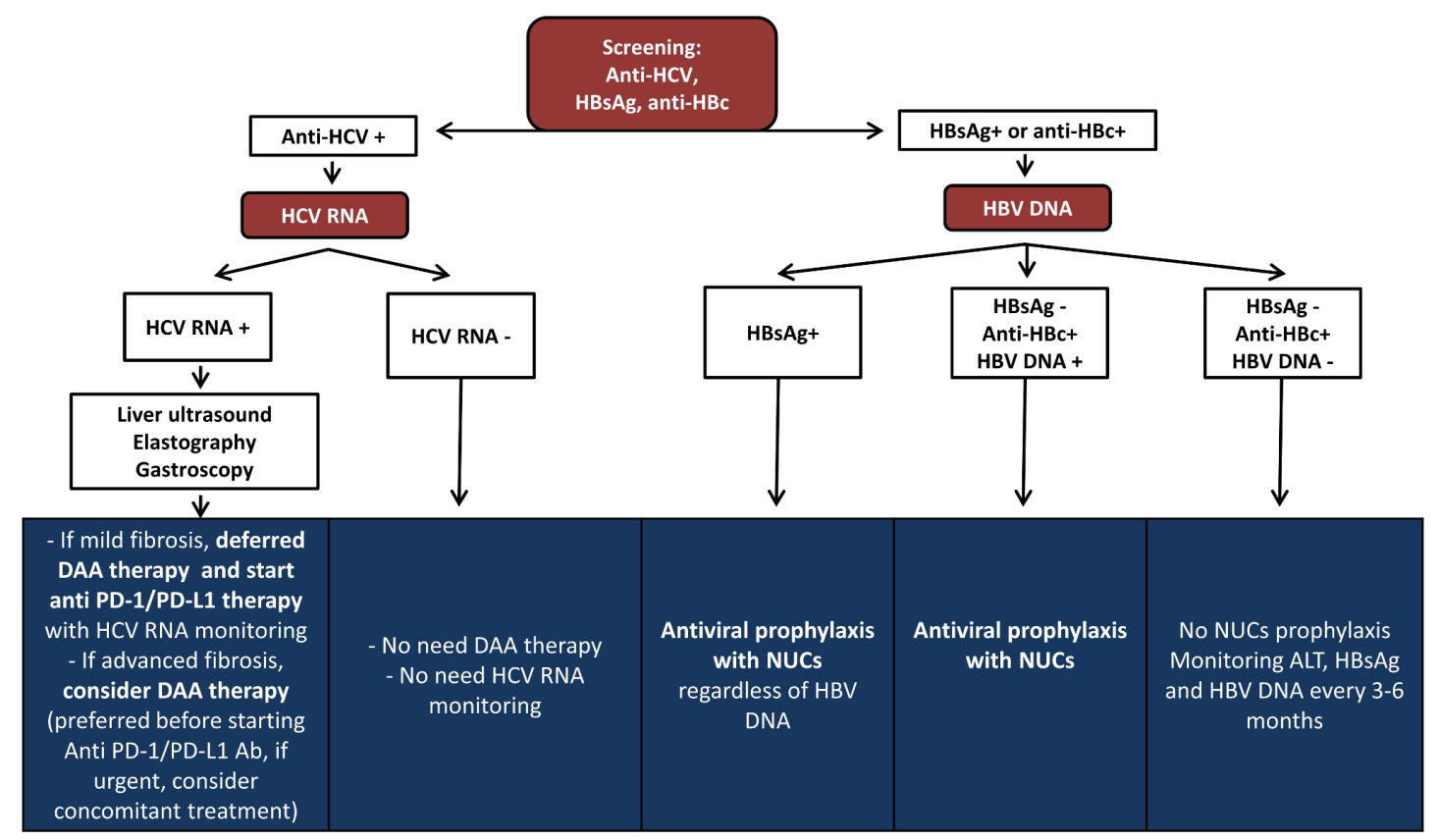

Figure 2 Diagram of clinical management of viral hepatitis-infected patient candidates for immunotherapy. ALT, alanine aminotransferase blood levels; DAA, direct-acting antiviral agents; HBsAg, hepatitis B surface antigen; HBV, hepatitis B virus; $\mathrm{HCV}$, hepatitis C virus; NUCs, nucleoside or nucleotide analogs; PD-1, programmed cell death-1; PD-L1, programmed cell death-1 ligand. 
Screening of HBV infection is based on the performance of both HBsAg and anti-HBc. In patients with a resolved $\mathrm{HBV}$ infection, there is a potential risk of viral reactivation, ${ }^{24}$ especially in rare cases that have an 'occult HBV infection' (anti-HBc-positive with detectable HBV DNA). Patients with $\mathrm{HBV}$ infection with positive $\mathrm{HBsAg}$ or were HBsAg-negative, and anti-HBc-positive with detectable HBV DNA, should be treated with NUCs before starting cancer immunotherapy (level of evidence III, grade of recommendation $\mathrm{A}$ ). In case of patients with negative HBsAg and undetectable HBV DNA in plasma, but positive anti-HBc, a close monitoring of alanine aminotransferase blood levels (ALT) levels, HBsAg and HBV DNA should be performed every 6 months during cancer immunotherapy (level of evidence III, grade of recommendation B). In case of ALT increment during immunotherapy, the first step should be urgent determination of both HBsAg and HBV DNA. When both are negative, the recommendation will be the same as that recommended for anti-HBc-negative subjects, although NUC prophylactic therapy should be discussed in the case of immunerelated adverse events development and a high dose of immune-suppressive drugs is needed (figure 2).

In patients following immunotherapy tretment who have no evidence of previous exposure to $\mathrm{HBV}$ and are unvaccinated (HBsAg negative/anti-HBc-negative/antiHBs-negative), there are no available data on the effectiveness or safety of $\mathrm{HBV}$ vaccination, so there is not enough evidence for or against vaccination recommendation. The panel recommends no prophylactic HBV vaccination immunotherapy in persons without high risk of infection.

In patients with HCV diagnosis, DAAs are indicated, delaying anti-PD-1/PD-L1 treatment until viral suppression is achieved (level of evidence III, grade of recommendation B). In cases where anticancer treatment must be started urgently, a simultaneous initiation of treatment with DAA could be considered (level of evidence $\mathrm{V}$, grade of recommendation $\mathrm{C}$ ) (figure 2).

\section{TB INFECTION}

\section{Incidence and pathophysiology}

TB is a global health challenge, now the second leading infectious cause of death in the world. Globally, in 2018, the incidence was 10 million people, and approximately $9 \%$ are persons living with HIV infection. There were an estimated 1.2 million TB deaths among HIV-negative people and an additional 251,000 deaths among HIV-1infected persons. In Europe, the estimated percentage of people infected by TB is around $3 \%{ }^{27}$ (online supplemental data 2). Preventive therapy with isoniazid for latent TB was introduced 20 years ago. Older studies precluded a fatality rate due to severe hepatotoxicity in $1 \%-19 \%$ of patients, but more recent studies, limiting the chemoprophylaxis to persons under 35 years old, have demonstrated a rate of clinical hepatotoxicity of only 1

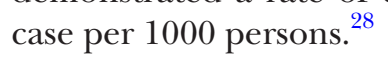

In patients with cancer, $\mathrm{TB}$ could be difficult to identify since the most frequent symptoms (cough, night sweats, fever and weight loss) and interstitial infiltrates in the lung are similar to those produced by cancer. ${ }^{29}$

Recent data have suggested that treatment with checkpoint inhibitors could reactivate latent $\mathrm{TB}$, although few cases of TB reactivation by anti-PD-1/PD-L1 antibodies have been reported and the real incidence of reactivations during immunotherapy is unknown (online supplemental table S1). ${ }^{29-31}$ On the other hand, other therapies that are commonly used for toxicity management of checkpoint inhibitors, such as anti-TNF antibodies, have a higher risk of TB reactivation. ${ }^{29} 3233$

\section{Assessment and diagnosis}

Mantoux tuberculin skin test (TST) or interferon gamma release assay (IGRA) is recommended before to immune checkpoint blockade administration (level of evidence $\mathrm{V}$, grade of recommendation C). ${ }^{34}$ TST is the standard method for determining whether a person is infected with Mycobacterium tuberculosis (MT). However, TST has sensitivity problems, with poor or non-existent response in patients with impaired cellular immunity, and also low specificity, given that some antigens are shared by nontuberculosis mycobacteria (NTM) and BCG vaccines previously inoculated. IGRA is a blood test that works by measuring the immune response to the TB bacteria. An IGRA test is not reactive to BCG vaccine or most NTM, so IGRA is more sensitive than TST in immunosuppressed individuals, more specific in the BCG vaccinated population, and in people infected with NTM. For patients with cancer, IGRA is preferred over TST. Both techniques demonstrate an immune response to MT antigens, rather than a true infection by the micro-organism. All positive patients must be evaluated to rule out active TB (level of evidence IV, grade of recommendation A). The evaluation includes clinical history, physical examination, and chest X-radiograph (or thoracic CT scan) and respiratory sampling. Patients with relevant clinical manifestations and/or abnormal chest X-radiograph should submit three sputum specimens (obtained via cough or induction at least 8 hours apart and including at least one early morning specimen) for acid-fast bacilli smear, mycobacterial culture, and nucleic acid amplification testing. Of these tests, the more sensitive are the molecular techniques that analyze the nucleic acid amplification, such as Xpert MTB/RIF or first-line and second-line Line probe assays (LPA). All unexpected suspicious lung lesions depicted by imaging should be investigated or biopsied, if possible. ${ }^{29}$ Importantly, in addition to TB reactivation, the differential diagnosis in such situations should always include metastatic progressive disease and granulomatous sarcoid-like reactions during cancer immunotherapy.

\section{Management and monitoring}

Patients with an IGRA positive test without diagnosis of active TB could be considered for TB chemoprophylaxis before starting immunotherapy (level of evidence $\mathrm{V}$, 
grade of recommendation C). Data available regarding chemoprophylaxis in patients with cancer immunotherapy are scarce, and this therapeutic strategy is based on data from patients treated with anti-TNF- $\alpha$ antibodies. Treatment during 4 weeks with isoniazid (plus rifapentine) significantly reduces the risk of developing active TB. ${ }^{35}$ Most studies suggest that treatment with isoniazid (plus rifapentine) should be initiated at least 1 month before anti-TNF- $\alpha$ administration, although it could be initiated at any time, even concurrent with anti-TNF- $\alpha$ administration. When a serious immune-related adverse event occurs, delaying anti-TNF- $\alpha$ is not reasonable. The main concern about chemoprophylaxis in patients who must complete immunotherapy is that isoniazid is hepatotoxic and it can increase the risk of immune-related liver toxicity. Moreover, when anti-PD-1/PD-L1 therapy is used in the adjuvant setting, delaying therapy until completion of chemoprophylaxis could be reasonable. However, in the advanced setting, recommendation to delay cancer treatment must be taken with caution. The panel cannot make a formal recommendation about chemoprophylaxis during immunotherapy, and the risk of hepatotoxicity must be balanced considering the low frequency of TB reactivation reported in the literature. Chemoprophylaxis could be mainly considered for young patients before adjuvant treatment, but in patients with advanced tumors, mainly older persons, delaying immunotherapy or assuming a high risk of toxicity starting chemoprophylaxis in combination with immunotherapy is not justified (level of evidence $\mathrm{V}$, grade of recommendation C). Patients who have initiated chemoprophylaxis should be monitored for clinical signs of hepatitis during TB chemoprophylaxis (level of evidence II, grade of recommendation A). In patients with hepatotoxicity due to chemoprophylaxis, chemoprophylaxis should be permanently discontinued, while immunotherapy should be reinitiated when hepatotoxicity is resolved (figure 3).

In case of active TB or TB reactivation during immunotherapy, immunotherapy must be withheld due to the possibility of an exaggerated inflammatory response (level of evidence IV, grade of recommendation A). Treatment should be started with high-dose corticosteroids and combination of isoniazid, rifampicin, pyrazinamide, and ethambutol for 8 weeks, followed by isoniazid and rifampicin for 18 weeks (level of evidence II, grade of recommendation A) (figure 3). There is no evidence about the exact time for resumption of immunotherapy after initiation of anti-TB treatment or if the immunotherapy must be permanently stopped. Several factors should be considered, including the severity of TB infection, overall tumor burden, tumor control, and comorbidities. In case of adjuvant immunotherapy, completion of anti-TB therapy should be the preferred option (level of evidence IV, grade of recommendation B) (figure 3). In case of immunotherapy for advanced cancer, a short course of TB therapy of at least 2 weeks is recommended before restarting cancer immunotherapy (level of evidence $\mathrm{V}$, grade of recommendation $\mathrm{C}$ ). In case of restarting immunotherapy, close monitoring is mandatory in order to check for overlapping toxicities, especially liver toxicities (level of evidence $\mathrm{V}$, grade of recommendation $\mathrm{C}$ ) (figure 3).

\section{SOLID TRANSPLANT RECIPIENTS Incidence and pathophysiology}

Long-term survival after solid organ transplantation has increased during the last decades. Solid organ transplant recipients (SOTRs) require lifelong immunosuppression. The required level of immunosuppression varies between different organ transplant types, with liver transplant being the one needing the lowest level. Calcineurin inhibitors, ciclosporin and tacrolimus, blocking signal 2 of $\mathrm{T}$ cell

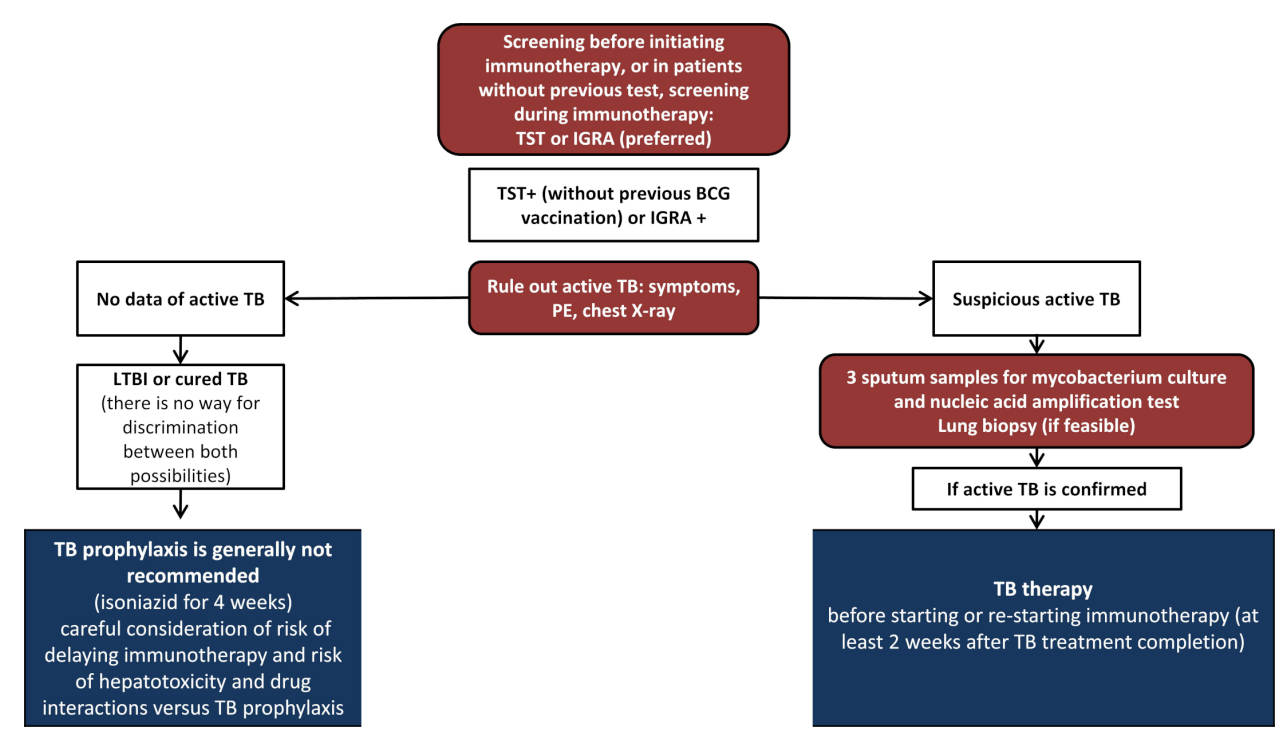

Figure 3 Diagram of TB screening and treatment of patient candidates for immunotherapy. IGRA, interferon gamma release assay; IO, Immunotherapy; LTBI, latent tuberculosis; PD-1, programmed cell death-1; PD-L1, programmed cell death-1 ligand; PE, physical examination; TB, tuberculosis; TST, Mantoux tuberculin skin test. 
activation are the main immunosuppressive medications used in combination with agents that interact with the cell cycle, such as mycophenolate mofetil. It is unclear whether these drugs interfere with the antitumor effect of anticancer immunotherapy, but SOTRs who develop malignant tumors pose a challenge for anticancer immunotherapy because $\mathrm{T}$ cell stimulation is implicated as one of the major mediators of allograft rejection.

The incidence of de novo malignancies, including nonmelanoma skin cancer, malignant melanoma, lymphoma, kidney, head and neck cancer, colorectal cancer, and lung cancer, is high in organ transplant recipients. These patients have been excluded from clinical trials with immune checkpoint inhibitors and the benefit and risks of such treatment have not been systematically evaluated in SOTRs. ${ }^{36}$ Serious immune-related adverse events seem to be as common as in the general population, although they are more frequent in patients without graft rejection. The main concern in these patients is the risk of graft rejection that occurs in $37 \%-41 \%$ of patients, with graft loss in almost $80 \%$ of cases and death due to graft rejection in $38 \%-45 \% .{ }^{37-41}$ Published data demonstrated no association with the length of time after organ transplantation and the risk of organ rejection. Although the rates of rejection are similar according to the different organs transplanted, analysis of case reports including data from 57 cases suggested that the rates of death secondary to graft rejection were higher in liver transplant patients $(30 \%)$ than kidney $(6 \%)$ or heart transplant $(0 \%)$ patients. ${ }^{38}$ Tumor response rate with anti-PD-1/PD-L1 or with anti-CTLA-4 antibodies varies between $25 \%$ and $40 \%$, depending on cancer histology subtype (online supplemental table S1). ${ }^{37-41}$ According to immunosuppressant medication, patients receiving low doses of prednisone $(\leq 10 \mathrm{mg}$ / day) seemed to have numerically higher tumor responses to immunotherapy with check point inhibitors therapy than those receiving mechanistic Target of Rapamycin (mTOR) inhibitors, calcineurin, or combination immunosuppressant therapy (63\% compared with $42 \%$ ), but in some series they also have a higher risk of graft rejection $(78 \%$ vs $11 \%),{ }^{37}$ whereas this has not been confirmed by others. ${ }^{38}$ So it is clear that SOTRs have a high allograft rejection rate that happens soon after immunotherapy initiation, with a high mortality rate. ${ }^{37-41}$ Immunotherapy reinitiation can be considered in patients who survived after graft rejection, supported by some case reports of treatment reinitiation in patients dependent on hemodialysis. ${ }^{4-44}$ Close collaboration between specialists is required to ensure an optimal patient management. Once the treating physician has determined that immunotherapy is the best option, the patient should always be informed about the risks to allow a shared decision.

\section{Management and monitoring}

There is no absolute contraindication for SOTRs with cancer to be treated with immunotherapy. The physician should raise a thorough discussion about the expected benefits and risks with the patient. Especially, treatment of patients with renal allograft must be considered. Patients' characteristics, the probability of achieving long-term survival, and alternative anticancer therapies should be considered (level of evidence $\mathrm{V}$, grade of recommendation $\mathrm{A}$ ).

When cancer immunotherapy is used in SOTRs, periodic examinations are required to closely monitor symptoms and serum levels of immunosuppressant drugs and to periodically adjust immunosuppressant drug doses or make drug modifications (level of evidence $\mathrm{V}$, grade of recommendation $\mathrm{C}$ ).

If graft rejection occurs, high-dose corticosteroids or other immunosuppressive therapies are used. In case of renal transplant, dialysis is usually required (level of evidence $\mathrm{V}$, grade of recommendation A). Cancer immunotherapy must be withheld in these cases until full recovery (level of evidence $\mathrm{V}$, grade of recommendation A). Immunotherapy reinitiation can be considered in patients who recover after graft rejection, especially in cases with renal transplant, who can survive in case of a graft loss (level of evidence $\mathrm{V}$, grade of recommendation C) (figure 4).

\section{OTHER CHALLENGING POPULATIONS: ALLOGENEIC TRANSPLANT, PREGNANCY AND AUTOIMMUNE DISORDERS Incidence and pathophysiology}

Other clinical challenging scenarios for treatment with checkpoint inhibitors include autoimmune disorders, cancer during pregnancy, and allogeneic transplant recipients (online supplemental table S1).

Treatment with anti-PD-1 antibodies yields a high rate of response in Hodgkin's lymphoma, but most patients will also be candidates for hematopoietic cell transplantation. Allogeneic bone marrow transplantation in patients previously treated with anti-PD-1 antibodies has a high risk of complications, mainly due to graft-versus-host disease, yielding high rates of antitumoral responses due to a simultaneous graft versus lymphoma effect. ${ }^{45}$ Similarly, anti-PD-1 therapy after allogeneic bone marrow transplantation has a high risk of graft-versus-host disease and immune-related toxicities, but it could be considered for patients with no other treatment options options. ${ }^{45}$

Although cancer during pregnancy is uncommon, when it occurs, is not unusual that immunotherapy with anti-PD-1 or anti-CTLA-4 antibodies is indicated, because one of the most frequent tumor during pregnancy is melanoma. In order for the fetus to not be rejected by the immune system of the mother, there is a state of immune tolerance during pregnancy. Theoretically, the inhibition of PD-1/PD-L1 or CTLA-4 during pregnancy will increase the risk of abortion and premature delivery, although three case reports in the literature have reported treatment with nivolumab, or in combination with ipilimumab, without relevant obstetric complications. ${ }^{46-48}$ These drugs probably do not increase the risk of malformations, but data on their teratogenic potential in humans are lacking.

Autoimmune disorders increase the risk of cancer development, including melanoma, so they produce a stage of chronic inflammation. One would expect that patients with autoimmune diseases would have an increased risk of immunerelated adverse events in case they are treated with anti-PD-1/ PD-L1 or anti-CTLA-4 antibodies. Several retrospective series 


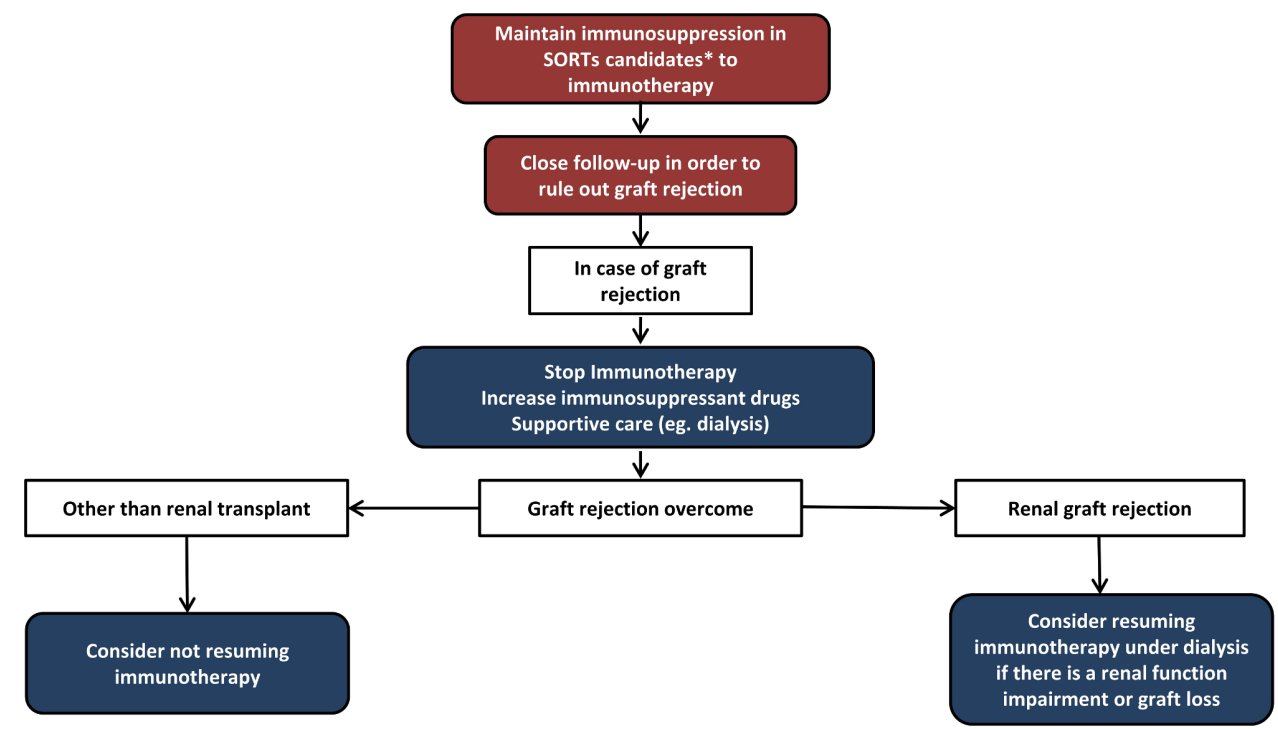

Figure 4 Diagram of immunotherapy management of patients with previous solid organ transplants. *Consider immunotherapy for patients with cancer histologies with high chances of responding to immunotherapy and in patients with good PS and no other comorbidities. PS, performance status; SOTRs, solid organ transplant recipients.

have presented data on the safety and activity of immunotherapy in patients with autoimmune disorders. ${ }^{40} \mathrm{In}$ summary, flare-ups of the pre-existing autoimmune disease were reported in $27 \%-50 \%$ of cases, including $10 \%-25 \%$ of high grade; moreover, $25 \%-30 \%$ of cases presented other immune-related adverse events, although a high response rate is suggested in this population. ${ }^{49} 50$

\section{Management and monitoring}

Previous treatment with allogeneic bone marrow transplantation is not an absolute contraindication to treatment with anti-PD-1 antibodies, and the same is true for patients previously treated with anti-PD-1 antibodies that are candidates for allogeneic bone marrow transplantation (level of evidence $\mathrm{V}$, grade of recommendation $\mathrm{A}$ ).

Pregnancy during treatment with immunotherapy must be avoided (level of evidence $\mathrm{V}$, grade of recommendation $\mathrm{A}$ ). In pregnant patients, treatment with checkpoint inhibitors is not absolutely contraindicated, although the risk of pregnancy complications is increased (level of evidence $\mathrm{V}$, grade of recommendation A).

In patients with a pre-existing autoimmunity, immunotherapy is not an absolute contraindication, but every case must be individually evaluated (level of evidence V, grade of recommendation A).

\footnotetext{
Author affiliations

${ }^{1}$ Translational Cancer Research Unit, Instituto Oncologico Dr Rosell, Dexeus University Hospital, Barcelona, Spain

${ }^{2}$ Oncology Department, Hospital Universitario Miguel Servet, Zaragoza, Aragón, Spain

${ }^{3}$ Liver Unit, Department of Internal Medicine, Vall d'Hebron Hospital Universitari, Barcelona, Catalunya, Spain

${ }^{4}$ Oncology Department, Vall d'Hebron Hospital Universitari, Barcelona, Catalunya, Spain

5 IrsiCaixa AIDS Research Institute, Badalona, Catalunya, Spain

${ }^{6}$ Department of Infectious Diseases, Hospital Universitari Germans Trias i Pujol, Badalona, Catalunya, Spain
}

${ }^{7}$ Department of Infectious Diseases, Hospital Universitari de Bellvitge, L'Hospitalet de Llobregat, Catalunya, Spain

${ }^{8}$ Oncology Department, Hospital Germans Trias i Pujol, Badalona, Spain

${ }^{9}$ Dermatology Department, Melanoma Group IDIBAPS, Hospital Clinic de Barcelona, Barcelona, Catalunya, Spain

${ }^{10}$ Oncology Department, Azienda 0spedaliero Careggi, Firenze, Toscana, Italy

${ }^{11}$ Oncology Department, Hospital Universitario La Paz, Madrid, Spain

${ }^{12}$ Catalan Institution for Research and Advanced Studies (ICREA), Catalan Institution for Research and Advanced Studies, Barcelona, Catalunya, Spain

${ }^{13}$ Oncology Department, Hospital Virgen de la Arrixaca, El Palmar, Murcia, Spain

${ }^{14}$ Oncology Department, Hospital Ramon y Cajal, Madrid, Spain

${ }^{15}$ Oncology Department, Hospital General Universitario Gregorio Marañón, Madrid, Spain

${ }^{16}$ Oncology Department, ASST Papa Giovanni XXIII, Bergamo, Italy

${ }^{17}$ Oncology Department, Consorci Hospital General Universitari de Valencia,

Valencia, Comunitat Valenciana, Spain

Correction notice This paper has been updated to correct author name 'Ivan Marquez- Rodas'.

Twitter Teresa Puertolas @TeresaPuertolas and Cristina Carrera @CarreraCris Acknowledgements The authors thank Stephanie Davis for revision of English language.

Contributors MG-C had full access to all the data in the study and took responsibility for the integrity and the accuracy of guidelines. Concept and design: MG-C, TP, MR, EM-C, RP, DP, EE, JM-P, IM and MM. Acquisition, analysis, or interpretation of data: MG-C, TP, MR, EM-C, RP, DP, EE, JM-P, CC, JLM, BR, JM, AB and MM. Drafting of the manuscript: MG-C, TP, MR, EM-C, CO, RP, DP, EE, IM, JM-P and MM. Critical revision of the manuscript for important intellectual content: MG-C TP, MR, EM-C, RP, DP, EE, JM-P, CC, JLM, BR, JM, CO, SF, BC, PC, AS, AB and MM. Obtained funding: $M G-C, E E$, and $A B$. Administrative, technical, or material support: $M G-C, E E$, and $A B$. Supervision: MG-C, EE, JM-P, IM and MM.

Funding This work was supported by the Spanish Melanoma Group (GEM). Competing interests None declared.

Patient consent for publication Not required.

Provenance and peer review Not commissioned; externally peer reviewed.

Open access This is an open access article distributed in accordance with the Creative Commons Attribution Non Commercial (CC BY-NC 4.0) license, which permits others to distribute, remix, adapt, build upon this work noncommercially, and license their derivative works on different terms, provided the original work is properly cited, appropriate credit is given, any changes made 
indicated, and the use is non-commercial. See http://creativecommons.org/ licenses/by-nc/4.0/

\section{ORCID iDs}

Maria Gonzalez-Cao http://orcid.org/0000-0003-3791-540X

Ivan Marquez-Rodas http://orcid.org/0000-0002-2476-668X

Mario Mandala http://orcid.org/0000-0001-8846-8959

\section{REFERENCES}

1 Mahale $\mathrm{P}$, Engels EA, Coghill AE, et al. Cancer risk in older persons living with human immunodeficiency virus infection in the United States. Clin Infect Dis 2018;67:50-7.

2 Vandenhende M-A, Roussillon C, Henard S, et al. Cancer-Related causes of death among HIV-infected patients in France in 2010: evolution since 2000. PLoS One 2015;10:e0129550.

3 Spano J-P, Veyri M, Gobert A. Immunotherapy for cancer in people living with HIV: safety with an efficacy signal from the series in real life experience. AIDS 2019;33:F13-19.

4 Puronen CE, Ford ES, Uldrick TS. Immunotherapy in people with HIV and cancer. Front Immunol 2019;10:10.

5 Gonzalez-Cao M, Morán T, Dalmau J, et al. Assessment of the feasibility and safety of Durvalumab for treatment of solid tumors in patients with HIV-1 infection: the phase 2 DURVAST study. JAMA Oncol 2020;6:1063.

6 Uldrick TS, Gonçalves PH, Abdul-Hay M, et al. Assessment of the safety of pembrolizumab in patients with HIV and advanced Cancer-A phase 1 study. JAMA Oncol 2019;5. doi:10.1001/ jamaoncol.2019.2244. [Epub ahead of print: 02 Jun 2019].

7 Cook MR, Kim C. Safety and efficacy of immune checkpoint inhibitor therapy in patients with HIV infection and advanced-stage cancer. JAMA Oncol 2019;5:1049-54

8 Gonzalez-Cao M, Martinez-Picado J, Karachaliou N, et al. Cancer immunotherapy of patients with HIV infection. Clin Trans/ Oncol 2019;21:713-20.

9 Blanch-Lombarte O, Gálvez C, Revollo B, et al. Enhancement of antiviral $\mathrm{CD}^{+} \mathrm{T}$-cell responses and complete remission of metastatic melanoma in an HIV-1-infected subject treated with pembrolizumab. JCM 2019;8. doi:10.3390/jcm8122089. [Epub ahead of print: 01 Dec 2019].

10 Gonzalez-Cao M, Martinez-Picado J, Rosell R. Safety of anti-PD-L1 inhibition in HIV-1-Infected patients with Cancer-Reply. JAMA Oncol 2020;6. doi:10.1001/jamaoncol.2020.3400. [Epub ahead of print: 10 Sep 2020].

11 Shah NJ, Al-Shbool G, Blackburn M, et al. Safety and efficacy of immune checkpoint inhibitors (ICIs) in cancer patients with HIV, hepatitis $\mathrm{B}$, or hepatitis $\mathrm{C}$ viral infection. J Immunother Cancer 2019;7:353.

12 Guihot A, Marcelin A-G, Massiani M-A, et al. Drastic decrease of the HIV reservoir in a patient treated with nivolumab for lung cancer. Ann Oncol 2018;29:517-8.

13 World Health Organization. Global hepatitis report, 2017. Available: https://www.who.int/hepatitis/publications/global-hepatitisreport2017/en/

14 Salleras L, Domínguez A, Bruguera M, et al. Declining prevalence of hepatitis B virus infection in Catalonia (Spain) 12 years after the introduction of universal vaccination. Vaccine 2007;25:8726-31.

15 Torres-Cornejo A, Lauer GM. Hurdles to the development of effective HBV immunotherapies and HCV vaccines. Pathog Immun 2017;2:102-25.

16 Carrat F, Fontaine H, Dorival C, et al. Clinical outcomes in patients with chronic hepatitis $\mathrm{C}$ after direct-acting antiviral treatment: a prospective cohort study Lancet 2019:393:1453-64.

17 El-Khoueiry AB, Sangro B, Yau T, et al. Nivolumab in patients with advanced hepatocellular carcinoma (CheckMate 040): an open-label, non-comparative, phase $1 / 2$ dose escalation and expansion trial. Lancet 2017;389:2492-502.

18 Zhu AX, Finn RS, Edeline J, et al. Pembrolizumab in patients with advanced hepatocellular carcinoma previously treated with sorafenib (KEYNOTE-224): a non-randomised, open-label phase 2 trial. Lancet Oncol 2018;19:940-52.

19 Wainberg ZA, Segal NH, Jaeger D, et al. Safety and clinical activity of durvalumab monotherapy in patients with hepatocellular carcinoma (HCC). JCO 2017;35:4071.

20 Rao N, Rizk EM, Hwang R, et al. Nivolumab for hepatocellular carcinoma (HCC) in a real-world context. J Clin Oncol 2019;37:e15675.

21 Zhang X, Zhou Y, Chen C, et al. Hepatitis B virus reactivation in cancer patients with positive hepatitis $B$ surface antigen undergoing PD-1 inhibition. J Immunother Cancer 2019;7:322.
22 Gane E, Verdon DJ, Brooks AE, et al. Anti-PD-1 blockade with nivolumab with and without therapeutic vaccination for virally suppressed chronic hepatitis B: a pilot study. $J$ Hepatol 2019;71:900-7.

23 Lee $\mathrm{P}-\mathrm{C}$, Chao $\mathrm{Y}$, Chen $\mathrm{M}-\mathrm{H}$, et al. Risk of HBV reactivation in patients with immune checkpoint inhibitor-treated unresectable hepatocellular carcinoma. J Immunother Cancer 2020;8:e001072.

24 Perrillo RP, Martin P, Lok AS. Preventing hepatitis B reactivation due to immunosuppressive drug treatments. JAMA 2015;313:1617-8.

25 Lake AC. Hepatitis B reactivation in a long-term nonprogressor due to nivolumab therapy. AIDS 2017;31:2115-8.

26 Pandey A, Ezemenari S, Liaukovich $\mathrm{M}$, et al. A rare case of Pembrolizumab-Induced reactivation of hepatitis B. Case Rep Oncol Med 2018;2018:5985131.

27 WHO global tuberculosis report, 2019. Available: https://www.who. int/tb/publications/global_report/en/

28 Nolan CM, Goldberg SV, Buskin SE. Hepatotoxicity associated with isoniazid preventive therapy: a 7-year survey from a public health tuberculosis clinic. JAMA 1999;281:1014-8.

29 Anastasopoulou A, Ziogas DC, Samarkos M, et al. Reactivation of tuberculosis in cancer patients following administration of immune checkpoint inhibitors: current evidence and clinical practice recommendations. J Immunother Cancer 2019;7:239.

30 van Eeden R, Rapoport BL, Smit T, et al. Tuberculosis infection in a patient treated with nivolumab for non-small cell lung cancer: case report and literature review. Front Oncol 2019;9:659.

31 Zaemes J, Kim C. Immune checkpoint inhibitor use and tuberculosis: a systematic review of the literature. Eur J Cancer 2020;132:168-75.

32 Langan EA, Graetz V, Allerheiligen J, et al. Immune checkpoint inhibitors and tuberculosis: an old disease in a new context. Lancet Oncol 2020;21:e55-65.

33 Kamboj M, Sepkowitz KA. The risk of tuberculosis in patients with cancer. Clin Infect Dis 2006;42:1592-5.

34 Champiat S, Lambotte O, Barreau E, et al. Management of immune checkpoint blockade dysimmune toxicities: a collaborative position paper. Ann Oncol 2016;27:559-74.

35 Swindells S, Ramchandani R, Gupta A, et al. One month of rifapentine plus isoniazid to prevent HIV-related tuberculosis. $N$ Engl J Med 2019;380:1001-11.

36 Engels EA, Pfeiffer RM, Fraumeni JF, et al. Spectrum of cancer risk among US solid organ transplant recipients. JAMA 2011;306:1891-901.

37 Abdel-Wahab N, Safa H, Abudayyeh A, et al. Checkpoint inhibitor therapy for cancer in solid organ transplantation recipients: an institutional experience and a systematic review of the literature. $J$ Immunother Cancer 2019;7:106.

38 Fisher J, Zeitouni N, Fan W, et al. Immune checkpoint inhibitor therapy in solid organ transplant recipients: a patient-centered systematic review. J Am Acad Dermatol 2020;82:1490-500.

39 Manohar S, Thongprayoon C, Cheungpasitporn W, et al. Systematic review of the safety of immune checkpoint inhibitors among kidney transplant patients. Kidney Int Rep 2020;5:149-58

40 d'Izarny-Gargas T, Durrbach A, Zaidan M. Efficacy and tolerance of immune checkpoint inhibitors in transplant patients with cancer: a systematic review. Am J Transplant 2020;20:2457-65.

41 Smedman TM, Line P-D, Guren TK, et al. Graft rejection after immune checkpoint inhibitor therapy in solid organ transplant recipients. Acta Oncol 2018;57:1414-8.

42 Ong M, Ibrahim AM, Bourassa-Blanchette S, et al. Antitumor activity of nivolumab on hemodialysis after renal allograft rejection. $J$ Immunother Cancer 2016;4:64.

43 Miller DM, Faulkner-Jones BE, Stone JR, et al. Complete pathologic response of metastatic cutaneous squamous cell carcinoma and allograft rejection after treatment with combination immune checkpoint blockade. JAAD Case Rep 2017;3:412-5.

44 Spain L, Higgins R, Gopalakrishnan K, et al. Acute renal allograft rejection after immune checkpoint inhibitor therapy for metastatic melanoma. Ann Oncol 2016;27:1135-7.

45 Herbaux C, Merryman R, Devine S, et al. Recommendations for managing PD-1 blockade in the context of allogeneic HCT in Hodgkin lymphoma: taming a necessary evil. Blood 2018;132:9-16.

46 Burotto M, Gormaz JG, Samtani S, et al. Viable pregnancy in a patient with metastatic melanoma treated with double checkpoint immunotherapy. Semin Oncol 2018;45:164-9.

$47 \mathrm{Xu} \mathrm{W}$, Moor RJ, Walpole ET, et al. Pregnancy with successful foetal and maternal outcome in a melanoma patient treated with nivolumab in the first trimester: case report and review of the literature. Melanoma Res 2019;29:333-7.

48 Mehta A, Kim KB, Minor DR. Case report of a pregnancy during ipilimumab therapy. J Glob Oncol 2018;4:1-3. 
49 Coureau M, Meert A-P, Berghmans T, et al. Efficacy and toxicity of immune -Checkpoint inhibitors in patients with preexisting autoimmune disorders. Front Med 2020;7:137.
50 Abdel-Wahab N, Shah M, Lopez-Olivo MA, et al. Use of immune checkpoint inhibitors in the treatment of patients with cancer and preexisting autoimmune disease: a systematic review. Ann Intern Med 2018;168:121-30. 


\section{Correction: Cancer immunotherapy in special challenging populations: recommendations of the advisory Committee of Spanish Melanoma Group (GEM)}

Gonzalez-Cao M, Puertolas T, Riveiro M for the Spanish Melanoma Group (GEM), et al. Cancer immunotherapy in special challenging populations: recommendations of the Advisory Committee of Spanish Melanoma Group (GEM). J Immunother Cancer 2021;9:e001664. doi: 10.1136/jitc-2020-00166

This paper has been updated to correct author name 'Ivan Marquez-Rodas'.

Open access This is an open access article distributed in accordance with the Creative Commons Attribution Non Commercial (CC BY-NC 4.0) license, which permits others to distribute, remix, adapt, build upon this work non-commercially, and license their derivative works on different terms, provided the original work is properly cited, appropriate credit is given, any changes made indicated, and the use is non-commercial. See http://creativecommons.org/licenses/by-nc/4.0/.

(C) Author(s) (or their employer(s)) 2021. Re-use permitted under CC BY-NC. No commercial re-use. See rights and permissions. Published by BMJ.

J Immunother Cancer 2022;10:e001664corr1. doi:10.1136/jitc-2020-001664corr1

D) Check for updates 